\title{
Advanced Structural and Chemical Analysis of Materials for Energy Related Applications
}

\author{
M.L. Trudeau*, A.M. Serventi*, K. Zaghib*, R. Veillette*, Lisa Rodrigue* and D. Thibault** \\ * Materials Science, Hydro-Québec Research Institute, 1800 Boul. Lionel-Boulet, Varennes, \\ Québec, Canada, J3X 1S1 \\ ** Mécanique, métallurgie et hydro-éolien, Hydro-Québec Research Institute, 1800 Boul. Lionel- \\ Boulet, Varennes, Québec, Canada, J3X 1S1
}

The enhanced industrial applications of nanostructured materials, as well as the need to understand the behaviour, and the degradation processes, of older materials with nanometer structures, necessitate an increased use of HRTEM for structural and, even more, for analytical analysis at the sub-nanometer level. This is even truer in the field of energy research today. For this reason, the Hydro-Quebec Research Institute has commissioned a combination of a HITACHI NB5000, nanoDUE'T focused ion beam and a Hitachi HD2700C dedicated STEM with a CEOS aberration corrector and a cold field emission gun, equipped with a specially designed EELS Spectrometer from Gatan and a newly designed SDD EDS detector from Bruker. This dedicated STEM has easily demonstrated an image resolution of $78 \mathrm{pm}$ in HAADF and an information resolution around $50 \mathrm{pm}$ in BF. Moreover, the unique combination of a cold emitter, a CEOS corrector and a new SDD detector allows for very fast EDS elemental analysis with the real possibility of elemental mapping at the nanometer or even sub-nanometer level. In this work, we will show the strategic importance of high resolution structural and chemical analysis for a variety of energy related research fields, from the synthesis of new electrodes for Li-ion batteries to the better understanding of more conventional materials such as martensitic stainless steel alloys used for water turbine to $\mathrm{Zr} 2.5 \mathrm{Nb}$ pressure tube alloy found in nuclear reactors.

For instance, the development of new materials for improved battery performances is the key to the mass usage of electrical vehicles. Since it well established today that these new materials need to be designed at the nanometer level, HR-STEM analysis is critical for understanding their properties as well as for IP consideration. Figure- $1 \mathrm{~b}$ presents a high resolution $\mathrm{BF}$ image of $\mathrm{LiFePO}_{4}$ crystals coated by a thin $\mathrm{C}$ layer. The presence of this $\mathrm{C}$ layer, in a very uniform way, is essential to increase the specific capacity of Li-ions batteries based on this material from 100-110 to $150-165 \mathrm{mAh} / \mathrm{g}$ [1].

On the other hand, for stainless steel alloys, used for the fabrication and repair of hydraulic turbine, it is critical to understand the deformation behaviour for different loading conditions. As fatigue is the predominant degradation mechanism, understanding the materials response to cyclic loading, at the nanometer level, especially at grain boundaries and along crack line, is at the basis of understanding the materials microscopic properties. Figure 2 a) presents a surface view of a microcrack developed during fatigue crack propagation experiments and b) the FIB lift-out taken around the crack tip. After thinning the lift-out down to around $40 \mathrm{~nm}$, observations of the alloy structure along the crack line in $\mathrm{BF}$ and $\mathrm{ZC}$, as presented in Figure-3, coupled to high-resolution EDS, should allow a correlation of the crack propagation mechanisms with the alloy nanostructure.

[1] The authors would like to acknowledge the assistance of M. Konno and D. Laul from Hitachi High Technologies for some samples observation. 

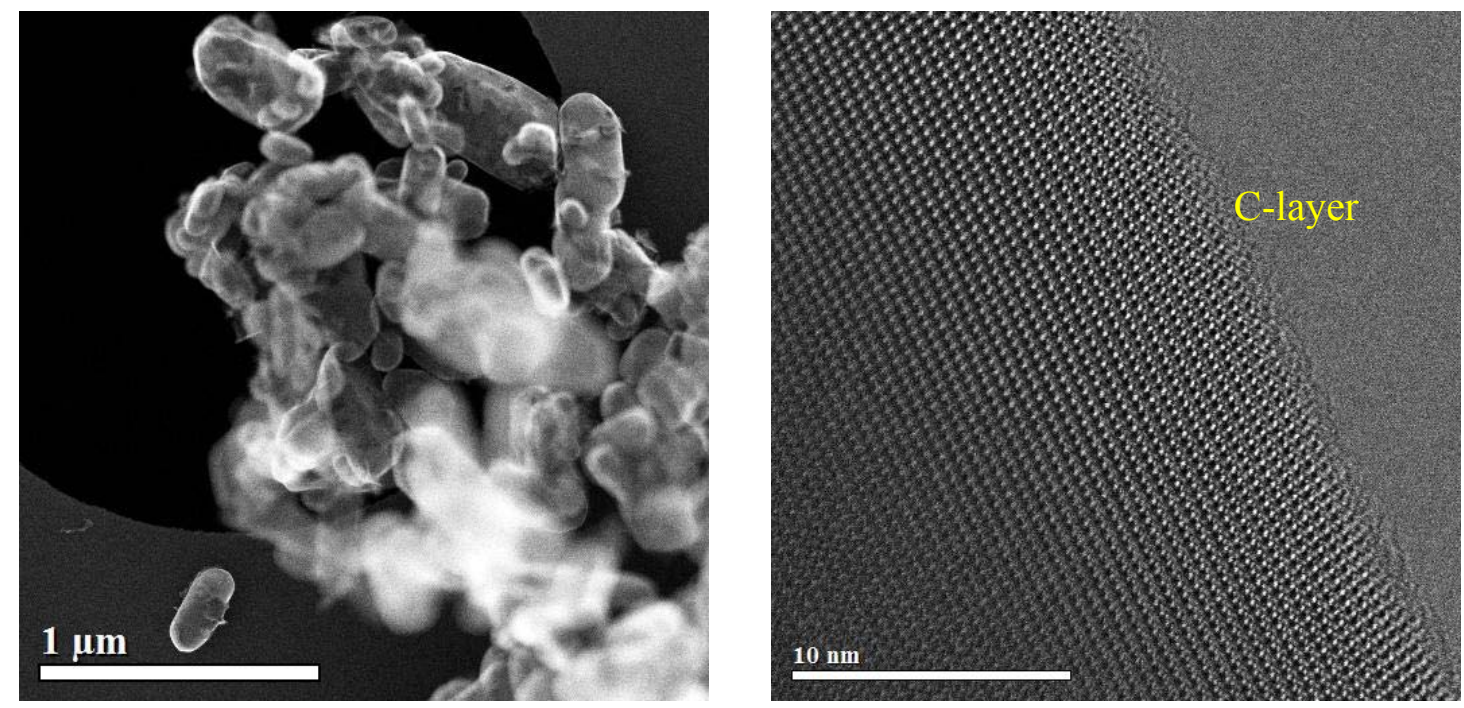

Fig. 1 C-coated $\mathrm{LiFePO}_{4}$ crystals a) SE image and b) High Resolution BF image showing the presence of a very thin $\mathrm{C}$ layer at the crystalline surface.
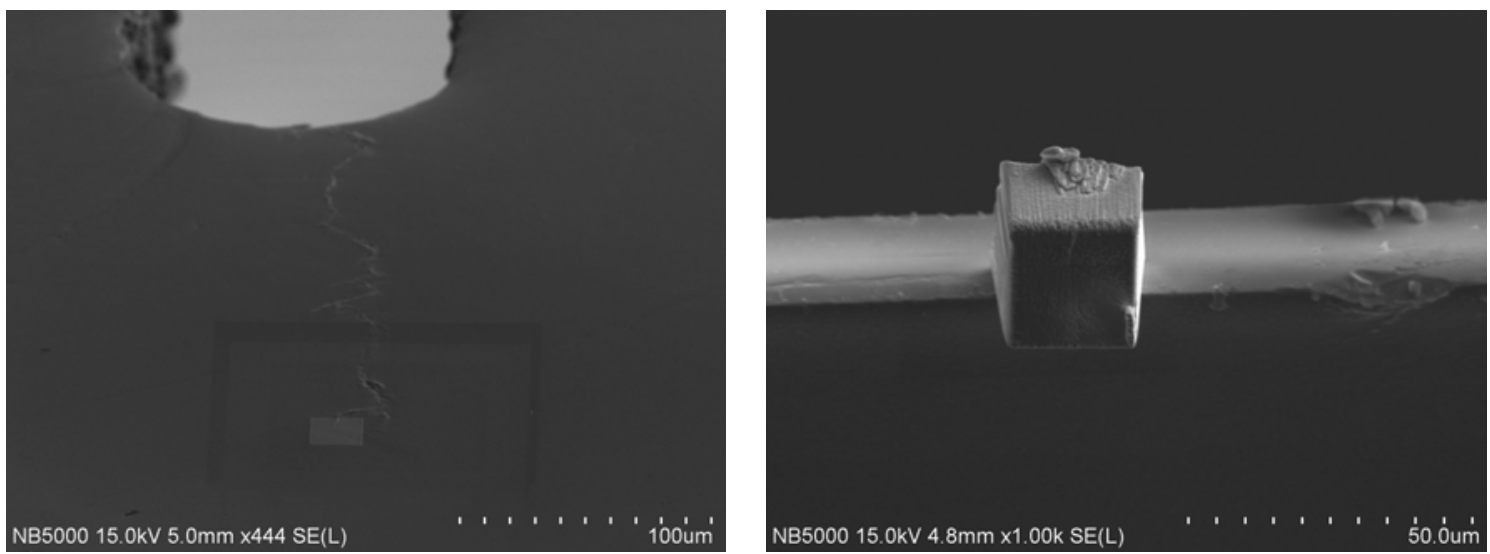

Fig. 2 SE image of a) a microcrack developed upon fatigue experiments in a martensitic stainless steel alloy and b) a FIB wedge taken directly at the tip of the crack.
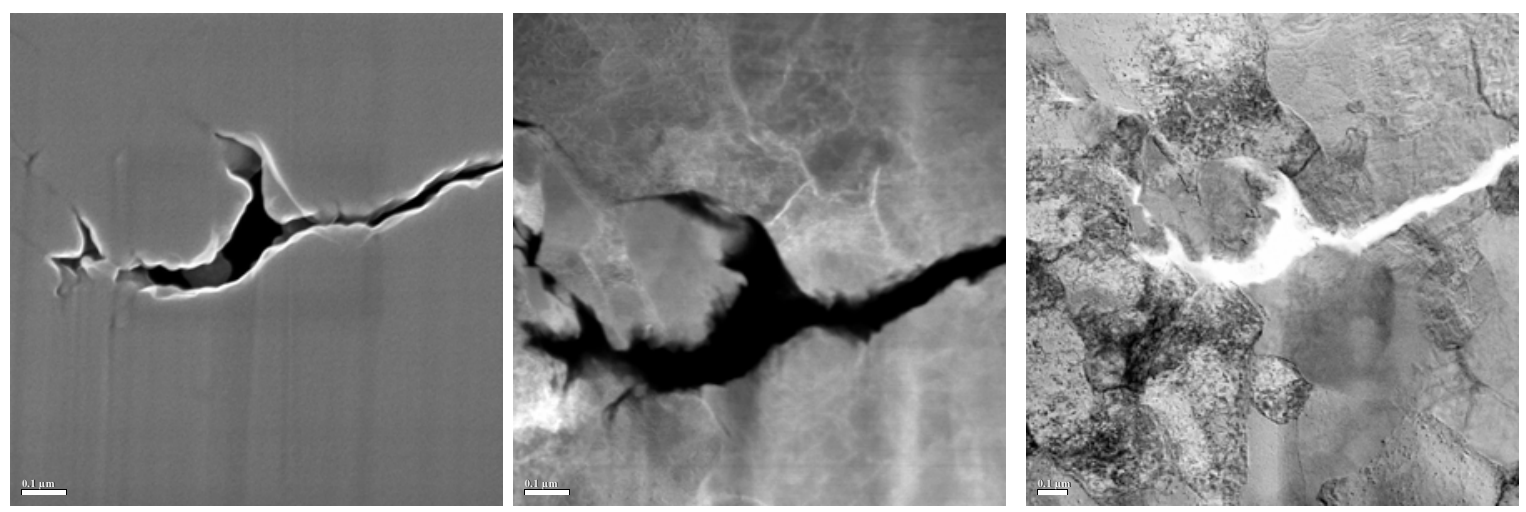

Fig. 3 a) SE b) ZC and c) BF micrographs of the crack tip. 